\title{
Intoxicación humana intencional por rodenticida anticoagulante de acción prolongada
}

\section{Intentional human poisoning by long-acting anticoagulant rodenticide}

\author{
Víctor Mechan-Méndez ', Julio Pedro \\ Ramírez-Erazo ${ }^{2}$, Diana Gonzales-Rafael ${ }^{3}$
}

Mechan-Méndez V, Ramírez-Erazo JP, Gonzales-Rafael D. Intoxicación humana intencional por rodenticida anticoagulante de acción prolongada. Rev Soc Peru Med Interna. 2021;34(2): 65-68.

https://doi.org/10.36393/spmi.v34i2.598

\begin{abstract}
RESUMEN
Varón de 19 años, que con fines suicidas ingirió tabletas de clonazepam y 250 pellets de un rodenticida conteniendo 2,5 mg de brodifacoum. Recibió cuidados generales y en los siguientes días presentó por hemorragias y prolongación del tiempo de protrombina (TP). Fue tratado con fitonadiona intravenosa y plasma fresco, observándose que los metabolitos cumarínicos urinarios desaparecieron después de dos meses y que el TP se normalizó después de nueve meses.
\end{abstract}

Palabras claves: rodenticida, anticoagulante de acción prolongada, superwarfarina, toxicidad, intento de suicidio, brodifacoum.

\section{ABSTRACT}

A 19-year-old male, who for suicidal purposes ingested clonazepam tablets and 250 pellets of a rodenticide containing $2.5 \mathrm{mg}$ of brodifacoum. He received general care and in the following days he presented bleeding and prolonged prothrombin time (PT). It was treated with intravenous phytonadione and fresh plasma, noting that urinary cumarin metabolites disappeared after two months and that PT was normalized after nine months.

Keywords: rodenticide, long-acting anticoagulant, superwarfarin, toxicity, suicide attempt, brodifacoum.

Médico hematólogo. Medicina Clínica-Hematología, Facultad de Medicina, Universidad Nacional Mayor de San Marcos, Lima-Perú. https://orcid.org/0000-0002-0178-5642

2 Médico internista. Facultad de Medicina, Universidad Nacional Mayor de San Marcos. Medicina Interna, Hospital Nacional Dos de Mayo, Lima-Perú.

https://orcid.org/0000-0002-5420-096X

3 Estudiante de medicina. Facultad de Medicina. Universidad Nacional Mayor de San Marcos, Lima-Perú.

https://orcid.org/0000-0002-006I-6433

\section{INTRODUCCIÓN}

La primera generación de rodenticidas anticoagulantes (RA), tipo warfarina, de corta duración, tuvo éxito en el control de roedores tras su aparición en 1948. Debido a la aparición de cepas resistentes de roedores se creó una segunda generación de hidroxi-cumarinas (superwarfarinas, RAAP), entre 1977 y 1992, con propiedades anticoagulantes de acción prolongada, que eliminan eficazmente roedores, ardillas y otros animales salvajes y que, dependiendo de la dosis, también pueden intoxicar a seres humanos. ${ }^{1}$

Nos referiremos en este articulo a la superwarfarina brodifacoum $\left(\mathrm{C}^{31} \mathrm{H}^{23} \mathrm{BrO}\right)^{3}$ presentada en pellets (comprimidos), pastas, mini bloques, bloques. ${ }^{2}$ Hasta inicios del año 2019, el brodifacoum era el rodenticida más comercializado en Perú.

Se conoce que la mayoría de las intoxicaciones en adultos asociadas a rodenticidas anticoagulantes de acción prolongada (RAAP) o superwarfarinas son accidentales, 92\% de casos; por ingestión intencional (suicidios), 4,8\%; y, homicidios, 3,4\%. ${ }^{1}$

Según información epidemiológica del Ministerio de Salud del Perú (MINSA), en el 2012, el Perú importó 3 829 toneladas métricas de rodenticidas, ocupando el segundo lugar en la lista total de pesticidas importados. 
Otro Boletín del MINSA, año 2015, reporto que, dentro de las intoxicaciones producidas por plaguicidas, solo el $6,2 \%$ había sido causado por rodenticidas tipo warfarinas, no mencionándose a las superwarfarinas. ${ }^{3}$ En el 2017, estudiantes universitarios reprodujeron los efectos hemorrágicos del ${ }^{\circledR}$ Klerat (brodifacoum), empleando pellets en ratas experimentales. ${ }^{2}$ En el Perú, la adquisición de superwarfarinas es de fácil acceso -sin receta- por regulación y conocimiento insuficientes sobre los riesgos de su ingesta accidental.

\section{PRESENTACIÓN DEL CASO}

Varón de 19 años, barman, natural y procedente de Yurimaguas, Departamento de Loreto. Con historia de depresión, desde noviembre del 2018, ingería fluoxetina por prescripción médica, tratamiento que abandonó a fines de enero del 2109. El 14/02/2019 se lanzó desde una motocicleta en movimiento autoinfligiéndose múltiples heridas, aunque no severas.

El 16/02/2019, ingirió a las 17.00 hs 40 tabletas de clonazepam de $0,5 \mathrm{mg}$ y 10 tabletas de $2 \mathrm{mg}$. Más tarde, a las $19.00 \mathrm{hs,} \mathrm{ingirió} \mathrm{abundantes} \mathrm{pellets} \mathrm{cubiertos} \mathrm{con}$ cereales conteniendo en total $2,5 \mathrm{mg}$ de brodifacoum, distribuidos en dos bolsas de $15 \mathrm{~g}$ (con 150 pellets en cada una, de los cuales ingirió 250), comercializados en el país con el nombre de Bin Laden ${ }^{\circledR}$, que lo sumieron en un sueño prolongado, por lo que fue trasladado a las $24.00 \mathrm{hs}$ del mismo día al hospital de Tarapoto, Departamento de San Martín, donde lo hidrataron y le realizaron un lavado gástrico, permaneciendo bajo observación, durante 10 días, al cabo de los cuales fue dado de alta, sin signos de sangrado el 27/02/2019.

A su regreso a Yurimaguas, el 28/02/2019, presentó hematuria, gingivorragia, epistaxis, hematomas en el tejido celular subcutáneo, cefalea, dolor corporal generalizado, ingresando al hospital de esta ciudad el 01/03/2019, donde le detectaron un valor de hemoglobina de $10,5 \mathrm{~g} /$ dl, Tiempo de Protrombina $>85$ seg. e INR entre 7,0 y 9,0 recibiendo por vía intravenosa $10 \mathrm{mg}$ de fitonadiona, una unidad de plasma y otra de sangre total, mejorando aunque persistiendo la hematuria y la prolongación del TP e INR, por lo que fue trasladado al Hospital Dos de Mayo de Lima, el 15/03/2019. En este hospital, el paciente fue tratado con ácido tranexámico $500 \mathrm{mg} /$ oral/cada 8 horas, alprazolam $0,5 \mathrm{mg}$ a las 8 p.m., risperidona $1 \mathrm{mg}$ a las 8 a.m., valproato de sodio $500 \mathrm{mg} /$ oral/cada 12 horas, fitonadiona $10 \mathrm{mg} /$ $\mathrm{ml}$ cada día y 14 unidades de plasma fresco. Con este tratamiento, el paciente mejoró desde el puinto de vista clínico y de laboratorio, tras cuatro meses de tratamiento.
22-03-2019: Rx de tórax normal. EEG y monioreo Holter normales.

04-08-2019: tomografía y resonancia magnética del cerebro con contraste: megacisterna magna (23 x $25 \mathrm{cc})$ asociada a ligera disminución en el tamaño del cerebelo. Ausencia de procesos inflamatorios o infecciosos, infartos o hemorragias de reciente instalación, resto del parénquima encefálico normal.

De los exámes de laboratorio, los recuentos de plaquetas y los dosajes de fibirnógenos, la bioquímica sanguínea y las pruebas uinmunológicas fueron normales.

Los metabolitos cumarínicos fueron detectados en muestras de orina durante los dos primeros meses de internamiento del paciente (Tabla 1), siendo las muestras sometidas a cromatografía en capa fina (CCF), las que tras adición de disolventes y adsorbentes específicos revelaron la estructura cumarínica evidenciada por la visibilizacion de una fuerte fluorescencia azul o verde a la luz UV, con valores Rf cercanos a $0,82 .{ }^{4}$ Los exámenes fueron realizados en el laboratorio CICOTOX (Centro de Informacion, Control Toxicologico y Apoyo Ambiental), Facultad de Farmacia y Bioquímica de la UNMSM.

Entre el segundo y tercer mes de tratamiento se detectaron dos recaídas clínicas y por los exámenes de laboratorio, superadas con ajustes del tratamiento. La hematuria desapareció en el cuarto mes de tratamiento. La normalización de los tiempos del TP y TTP fueron demostradas mediante pruebas de mezcla del plasma del paciente con plasma normal, constatándose déficit de factores de coagulación y no de inhibidores. Ver Tabla 1.

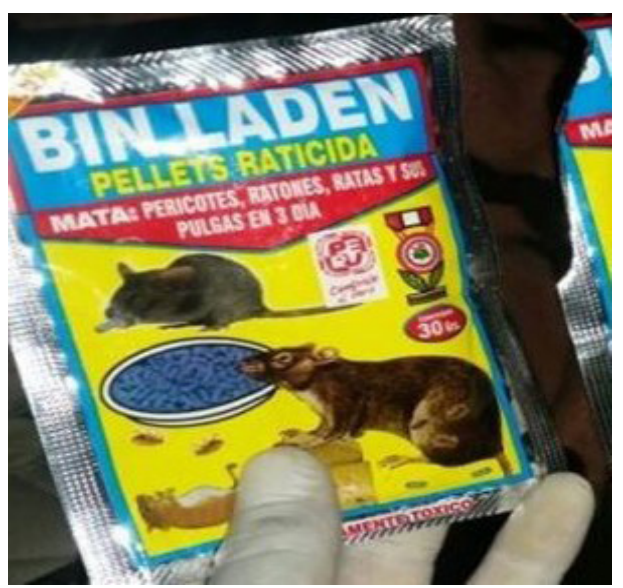

Figura I. Rodenticida Bin Laden.

\begin{tabular}{|l|l|l|l|l|l|l|}
\hline & \multicolumn{7}{|l|}{$\mathbf{N}^{\circ}$ de días post ingestión } \\
\hline & Valores normales & $\mathbf{2 8}$ & $\mathbf{3 6}$ & $\mathbf{5 2}$ & $\mathbf{5 9}$ & $\mathbf{8 3}$ \\
\hline TP (seg) & $10-14$ & 120,0 & 27,7 & 47,6 & 23,2 & 30,5 \\
\hline INR & $\mathrm{I}, 2-1,5$ & $\mathrm{II}, 50$ & 2,37 & 5,34 & 2,05 & 2,0 \\
\hline TTP (seg) & $25-35$ & 86,5 & 45,9 & 50,0 & $5 \mathrm{I}, 0$ & 50,5 \\
\hline Cumarinas en orina & & $(-)$ & $(+)$ & $(-)$ & $(+)$ & $(-)$ \\
\hline
\end{tabular}




\section{DISCUSIÓN}

Analizamos un síndrome hemorrágico asociado a prolongaciones persistentes del TP e INR, causados por la ingestión intencional de un rodenticida anticoagulante de accion prolongada (RAAP), promovido por la exacerbación de un estado depresivo crónico e inductor de un frustrado intento de suicidio. La alta afinidad del brodifacoum por la enzima Vitamina K (1)-2.3 epóxido reductasa permite la inhibición competitiva de la síntesis de vitamina $\mathrm{K} 1$ (factores: II, VII, IX, X y las proteínas C y S), no afectando a los factores sintetizados previamente, lo que explica el inicio tardío (3-7 días, raramente un día después) de las primeras manifestaciones hemorrágicas, enfatizándose que los alargamientos del TP e INR ocurren cuando los niveles de los factores II, VII, IX y $\mathrm{X}$ disminuyen a menos del $25 \%$ de sus valores normales. ${ }^{1}$

En el Perú, solo se expenden pellets conteniendo una de dos sustancias activas de RAAPs: el brodifacoum y la bromadialona, siendo el primero el más comercializado, manufacturado por compañías brasileñas y adquirido por sus pares peruanas, que las expenden bajo nombres exóticos como Bin Laden ${ }^{\circledR}$. Hasta principios del 2019, la cantidad de brodifacoum (principio activo), contenido en cada pellet de un RAAP era uniforme, 0,005\%, y acondicionadas en bolsas de $30 \mathrm{~g}$. conteniendo 150 pellets equivalentes a $1,5 \mathrm{mg}$ por cada bolsa o bloques de $20 \mathrm{~g}$ conteniendo $1 \mathrm{mg}$. del principio activo. ${ }^{2}$ En el presente caso, se constató la presencia de brodifacoum, cuyos metabolitos fueron identificados en la orina mediante CCF (Ver Tabla I), durante los dos primeros meses, pese al intenso tratamiento con plasma y el antídoto correspondiente. La presencia prolongada del brodifacoum fue favorecida por su metabolismo especial que le permite ser detectado en todos los tejidos y en los humores excretados por el cuerpo, descartándose la presencia de la bromadialona porque los dos metabolitos principales de esta sustancia se excretan fundamentalmente por las heces y escasamente por la orina $(<1 \%)$, apenas en los cuatro primeros días post ingestión de cantidades tóxicas. ${ }^{4}$

La principal diferencia entre los RAAPs y los rodenticidas anticoagulantes (RA), o warfarinas de primera generación, es que la eliminación de la vida media del compuesto activo de brodifacoum oscila entre 16 a 34 días, bastante prolongado debido a su alta solubilidad en lípidos, su gran afinidad por el tejido hepático, su eliminación de orden cero cuando las concentraciones de las RAAPs son muy altas y permanecer en la circulación enterohepática. No obstante, aunque el pico plasmático ocurre entre 2 a 8 horas después de la ingesta del rodenticida, los efectos clínicos hemorrágicos son observables entre el tercer y séptimo día post-ingestión, como sucedió en este paciente, porque el RAAP ingerido no afecta a los factores sintetizados antes de la ingesta del toxico. ${ }^{5}$

Clinicamente, es posible establecer una escala de gravedad de intoxicación por RAAPs. La grave supone presencia de hemorragias masivas (gastrointestinales, pulmonares o intracraneales) capaces de comprometer la vida de los intoxicados. ${ }^{6}$ La intoxicación moderada implica hemorragias que no ponen en riesgo la vida del paciente (equimosis, hematomas, hemartrosis, epistaxis, gingivorragias, hematoquecias, hematuria, menometrorragia, hematemesis, hemoptisis). Los casos leves se caracterizan por irritabilidad gastrointestinal y ausencia de sangrado macroscópico. ${ }^{7}$

En casos de intoxicación severa, la literatura aconseja utilizar el complejo de protrombina o el factor VII recombinante. Por el momento y según algunos investigadores, no existe un tratamiento estandarizado para las dosis de vitamina $\mathrm{K} 1$, por lo que estas deben ajustarse individualmente hasta obtener un INR $<2$. Aunque raros, los efectos secundarios de la vitamina K1 administrada a dosis altas ocurren en 1/ cada 10000 personas en forma de hipotensión, reacción alérgica grave y flebitis en el sitio de administración. ${ }^{6}$

La dosis tóxica mínima de brodifacoum es $\geq 1 \mathrm{mg}$, registrada en un paciente de 20 años que tenía ligero sangrado, sin riesgo vital. ${ }^{7}$ De otro lado, la toxicidad severa con riesgo de muerte se asocia a dosis de entre 4,5 a $24,0 \mathrm{mg}$ de brodifacoum, existiendo asimismo otro factor a considerar que es la ingestión crónica de bradifacoum. ${ }^{6}$

El tratamiento terapéutico con vitamina $\mathrm{K} 1$, en estos pacientes, solo está indicado en aquellos que tienen factores de riesgo asociado como haber ingerido al menos $1 \mathrm{mg}$ de brodifacoum o en aquellos que tienen un valor de INR $\geq 2$, aunque otros investigadores han propuesto valores de INR $\geq 4$, por indicar un incremento de 4 y 7 veces mas de riesgo de hemorragia intracerebral o subdural, respectivamente. La vitamina $\mathrm{K} 1$, en dosis de $10 \mathrm{mg} / \mathrm{ml}$, debe administrarse de manera exógena hasta que se elimine el anticoagulante, siendo la vía intravenosa la preferida para casos severos, pudiendo repetirse cada 6 a 8 horas, según sea necesario. Un error en nuestro paciente fue constatar que solo había recibido fitonadiona intravenosa una vez al día. Existen informes de empleo de vitamina K1 de entre 6 a 9 meses de duración. ${ }^{8}$

La dosis oral terapéuticamente efectiva de vitamina K1 es 7 $\mathrm{mg} / \mathrm{kg} / \mathrm{cada} 24$ horas, cada 6 horas. Algunos investigadores que estudiaron la toxicidad humana por RAAP, lograron medir las concentraciones séricas de bradifacoum en pacientes que habían suspendido el tratamiento previo con altas dosis orales de vitamina $\mathrm{K} 1$, porque tenían INR $\leq$ 2 , notando que 11 de ellos de un total de 21 pacientes, exhibieron concentraciones de bradifacoum por encima de un nivel seguro $(10 \mathrm{ng} / \mathrm{ml})$, causantes en algunos de ellos de recaídas, por lo que la normalización del INR, no sería un criterio suficiente para suspender la vitamina $\mathrm{K} 1$ oral, sino las concentraciones séricas de brodifacoum. ${ }^{9}$ En nuestro paciente se favoreció la administración de plasma fresco congelado dos veces por semana durante dos meses, hasta la normalización del TP, INR y desaparición del síndrome hemorrágico. Sin embargo, el plasma fresco no fue totalmente efectivo, porque nuestro paciente tuvo dos recaídas, clínica y de laboratorio, en el segundo y tercer mes, siendo dado de alta con la prescripccion de ingerir $10 \mathrm{mg}$ de fitonadiona oral cada/6 horas y regresar 
en dos meses, adjuntando los últimos valores de TP e INR, requerimiento que cumplió, al normalizar sus valores de TP e INR, tras nueve meses de tratamiento.

De otro lado y, si bien los familiares del paciente informaron no haber notado epistaxis o hematuria, durante su primera estadía de 10 días, en un hospital de la amazonia peruana, nos preguntabamos si el lavado gástrico repetido que recibió nuestro paciente ocho horas después de ingerir el RAAP y otros medicamentos (antidepresivos, benzodiacepinas), atenuó la gravedad de la intoxicación. La literatura revisada nos informa que la aparición de las primeras manifestaciones hemorrágicas ocurre entre 4 y 7 días, por razones explicadas anteriormente, después de la ingesta de RAAPs. ${ }^{1}$ Tocante al lavado gástrico, algunos investigadores argumentan que este procedimiento solo tiene validez terapéutica contra ingestas masivas ( $>4 \mathrm{mg}$ de brodifacoum), siempre que se realicen hasta una hora después de la ingestión del RAAP, empero, no existe evidencia que indique que esta medida reduzca su absorción. Alternativamente, la administración de carbón activado en dosis única, hasta una hora después de la ingestión del tóxico, logra, al menos in vitro, la adsorción del brodifacoum. ${ }^{10}$

Respecto a los síntomas depresivos, el paciente fue evaluado por las unidades de Psiquiatría y Psicología del hospital, se le diagnosticó de depresión crónica moderado, Recibiendo tratamiento y apoyo emocional.

\section{REFERENCIAS BIBLIOGRÁFICAS}

I. Feinstein DL, Akpa BS, Ayee MA, et al. The emerging threat of superwarfarins: history, detection, mechanisms, and countermeasures. Ann N Y Acad Sci. 2016;1374 (I):III-I22. doi: I0. I I I I/nyas. 13085

2. Informe Técnico. Klerat pellets. Compañía: Syngenta Proteçao de cultivos.Ltda., Brasil. Importador y distribuidor:Tecnología Química y Comercio S.A. Ate-Vitarte Perú. Visto el 07.07.2019. Disponible en: https://www.tqc.com.pe/wp-content/uploads/2020/08/Kleratpellets-Fichal-Tecnica.pdf

3. Boletin Epidemiológico, Ministerio de Salud del Perú. Situación epidemiológica de las intoxicaciones agudas por plaguicidas en el Perú. 20I5; 24 (39). 79I-795. URL disponible en: https://www.dge. gob.pe/portal/docs/vigilancia/boletines/2015/39.pdf
4. Grobosch T,Angelow B, Schönberg L, Lampe D.Acute bromadiolone intoxication.Acute bromadiolone intoxication.J.Anal Toxicol. 2006;30 (4):28I-6. doi: I0.1093/jat/30.4.28I

5. Hauck ZZ. Tissue distribution, pharmacokinetics and metabolism of brodifacoum: a superwarfarin. Dissertation submitted as partial fulfillment of the requirements for the degree of Doctor of Philosophy in Pharmacognosy in the Graduate College of the University of Illinois at Chicago, 2017. URL disponible en: file://I Users/Guest/Downloads/Hauck-Dissertaion-20I7.pdf

6. Wu YF, Chang CS, Chung CY, Lin HY, Wang CC, Shen MC. Superwarfarin intoxication: hematuria is a major clinical manifestation. Int J Hematol. 2009;90 (2): I70-3. doi: 10.1007/s I 2 I85009-0374-6

7. Caravatti EM, Erdman AR, Scharman EJ, Woolf AD, Chyka PA, Cobaugh DJ, Wax PM, Manoguerra AS, Christianson G, Nelson LS, Olson KR, Booze LL, Troutman WG. Long-acting anticoagulant rodenticide poisoning: an evidence-based consensus guideline for out-of-hospital management. Clin Toxicol (Phila). [en línea] 2007;45 (I): I-22. PMID: I 7357377. URL disponible en: https://www.ncbi.nlm. nih.gov/pubmed/I7357377

8. Park J. Can we more efficiently save patients with vitamin $\mathrm{K}$-dependent coagulopathy caused by superwarfarin intoxication?. Korean J Intern Med. 20I4; 29 (4): 430-433. doi: 10.3904/ kjim.20I4.29.4.430

9. Israel Rubinstein----Guy Weinberg, Richard van Breemen, Ronald C. Hershow \&Douglas L. Feinstein. Treatment for long acting anticoagulant rodenticide poisoning - beyond INR monitoring? Toxicology Communicactions. 2018; 2 (I), pp:59-6I. https://doi.org/I0.I 080/24734306.2018.1500152

10. Benson BE, Hoppu K., Troutman WG, Bedry R, Erdman A., Höjer J, Mégarbane B, Thanacoody R, Caravati EM. Position paper update: gastric lavage for gastrointestinal decontamination. Clin Toxicol. 201 3; 5I:|140. doi: I0.3109/|5563650.2013.770I54

\section{CORRESPONDENCIA:}

Víctor Mechán-Méndez

vmechanm@unmsm.edu.pe

Fecha de recepción: 28-02-2021.

Fecha de aceptación: 24-05-2021.

Financiamiento: por los autores.

Conflicto de interés: ninguno, según los autores. 